\title{
A Network Pharmacology Analysis to Reveal the Molecular Mechanism of Zhizi-Danshen on Coronary Heart Disease
}

\section{Yue-hong Shen}

Affiliated Hospital of Integrated Traditional Chinese and Western Medicine, Nanjing University of Chinese Medicine

\section{Shu-lin Wang}

Zhenjiang Hospital of Traditional Chinese Medicine

\section{$\mathrm{Na} \mathrm{Wu}$}

Affiliated Hospital of Integrated Traditional Chinese and Western Medicine, Nanjing University of Chinese Medicine

\section{Yu-chen Dai}

Nanjing University of Chinese Medicine

\section{Qian Zhou}

Affiliated Hospital of Integrated Traditional Chinese and Western Medicine, Nanjing University of Chinese Medicine Jian-ping Shen ( $\square$ Shenjp@jsatcm.com )

Nanjing University of Chinese Medicine

\section{Research}

Keywords: Network Pharmacology, Zhizi, Danshen, Molecular Mechanism, Coronary Heart Disease Posted Date: October 7th, 2020

DOl: https://doi.org/10.21203/rs.3.rs-85979/v1

License: (c) (i) This work is licensed under a Creative Commons Attribution 4.0 International License. Read Full License 


\section{Abstract}

Objective

Our study aimed to investigate the potential mechanisms of the herb pair Zhizi-Danshen (ZD) for coronary heart disease (CHD) using network pharmacological data mining technology.

Methods

The Traditional Chinese Medicine System Pharmacology (TCMSP) database was used to collect the active ingredients of ZD and predict ZD-related target proteins. Afterwards, we identified CHD-related targets from DisGeNET database, NCBI gene database, and TTD database. The common targets both from ZD and CHD were screened by Venny2.1, which were then imported into the String database for protein-protein interaction (PPI) analysis. Finally, the $\mathrm{GO}$ and KEGG enrichment analysis were performed by R software, and the network construction was established using Cytoscape3.7.2.

Results

We obtained 199 possible targets from 62 candidate ingredients of ZD and 1033 CHD-ralated targets, with 83 overlapping common target genes. Then, 11 core targets were acquired from PPI network analysis. Further, GO analysis showed that these common targets mainly influenced receptor ligand

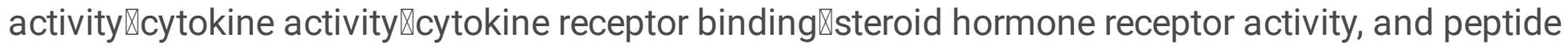
binding. KEGG pathway analysis indicated that ZD affected CHD through seven important pathways linked to vascular endothelial function regulation (fluid shear stress and atherosclerosis $\triangle A G E-R A G E$ signaling pathway in diabetic complications, HIF-1 signaling pathway), imflammatory effects (IL-17

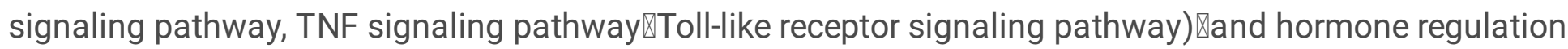
(relaxin signaling pathway).

Conclusions

This study revealed the potential pharmacological mechanisms of ZD against CHD, which were mainly associated with regulation of vascular endothelial function and inflammatory effects, promotion of vasodilatation, and prevention of cardiac fibrosis. Moreover, it provided a novel conception for the development of alternative therapies on CHD.

\section{Introduction}

Coronary heart disease (CHD) attracts global concern because of its high morbidity, mortality, and recurrence rate, accounting for the vast majority of cardiovascular events [1-2]. It originates from longstanding coronary atherosclerosis, with the clinical manifestations of angina, myocardial infarction, chronic arrhythmia, heart failure, and even sudden death [3-5]. Currently, western medicine is the main therapeutic way to prevent the progression of CHD. However, there still exists some minuses with modern medicine such as inevitable side effects, high drug cost, and multiple drugs taken as lifelong therapy [6- 
7]. Moreover, the hypothesis of "one drug, one target, one disease" is no longer applicable for chronic and complex diseases including CHD due to treatment restriction in recent years [8]. Thus, it is essential to seek multi-target and safe therapeutic interventions in order to improve the survival rate of CHD patients and reduce the social and economic burden.

It is true that Traditional Chinese Medicine (TCM), as a complementary and alternative therapy of modern medicine, has a unique curative effect in the treatment of various diseases [9]. Compared with the single target and high selectivity of Western medicine, TCM possesses the advantages of multi-component, multi-target, multi-pathway, and small side effects, which has always been the focus of new drug investigation [10-11]. According to Chinese medicine theory combined with modern research, "blood stasis" together with "heat toxin" are considered as important factors in the pathogenesis of CHD [12]. Chinese herbs Gardenia jasminoides ( Chinese name Zhizi) and Salvia miltiorrhiza ( Chinese name Danshen) both from the oldest Chinese book of material medica "Shennong Herbal Classic" have the effects of antifebrile and detoxifcatio, and activating blood circulation to dissipate blood stasis, thus being widely used in cardiovascular diseases [13-14]. Additionally, pharmacological studies have shown that Zhizi can imhibit inflammation, nourish the liver and gallbladder, protect endothelial cells, and prevent atherosclerosis [15-16]. And Danshen is often used as intravenous herbal medicine to treat angina pectoris and acute myocardial infarction owing to the functions of protecting myocardium, inhibiting platelet aggregation, lowering blood pressure, and performing antioxidation [17-18]. Further》 some active ingredients of Zhizi and Danshen have been shown with cardioprotective effects. For example, crocetin, the effective component of Zhizi, could reduce activity of serum creatine kinase (CK) and lactate dehydrogenase (LDH), and increase myocardial tissue ATP, thereby improving myocardial ischemia [19]. Salvianolate extracted from Danshen reduced myocardial infarction area by increasing activity of superoxide dismutase (SOD) and thioredoxin, raising glutathione concentration, and decreasing malondialdehyde (MDA) concentration [20]. Therefore, we believe that the herb pair ZhiziDanshen (ZD) is beneficial for the treatment of CHD. However, due to their complex chemical compounds, the molecular mechanism of anti-CHD remains unclear, which needs to be further elucidated.

Network pharmacology is an emerging approach to drug design integrating systems biology and network analysis, which promotes the transformation from "one target, one drug" model to a new "network target, multi-component therapy" model [21-22]. The core concept of network pharmacology that focuses on integrity and systematization coincides with holism in TCM, and the combination of both establishes a new method to TCM network pharmacology. Besides, it reveals the complicated interactions between Chinese herbs, organism, and diseases from the system perspective and molecular level by predicting the targeted distribution and pharmacological effects of TCM compounds [22-23]. In this study, we used network pharmacology approach to construct visualization network and comprehensively demonstrate the active ingredients and molecular details of ZD against CHD. The compounds of ZD and target proteins were identified from the network databases. The core targets were screened through PPI network, and the underlying mechanism of ZD acting on CHD was investigated in terms of molecular function and signaling pathways by enrichment analysis. The current study provided the pharmacological basis for the 
multi-component, multi-target and multi-pathway therapeutic effects of ZD against CHD, suggesting the possibility for clinical application and new drug research.

\section{Methods}

\section{Active Compound of ZD and Target Prediction}

The active compounds of ZD were acquired from Traditional Chinese Medicine System Pharmacology (TCMSP \http://tcmspw.com/tcmsp.php) database. This database [24] is a systematic pharmacology platform that reveals the mechanisms of TCM by identifying drug-target network and drug-disease network, and covers pharmacokinetic properties including absorption, distribution, metabolism, and excretion (ADME). The basic properties of chemical compounds such as molecular name, molecular weight(MW), oral bioavailability(OB), drug likeness(DL), and oil-water partition coefficient(AlgP) can be obtained from TCMSP database. Specifically, OB mainly affects the performance of drugs, which is the ability of drugs absorbed into the blood circulation [25]. DL describes the possibility of compounds to become drugs, and help to optimize pharmacokinetics and drug properties [26]. AlgP reflects the distribution of drugs in oil-water phases. The smaller the value, the better the water solubility is [27]. We consulted relevant literatures which suggested compounds of Zhizi with $\mathrm{OB} \geq 10 \%, \mathrm{DL} \geq 0.18, \mathrm{AlgP} \leq 5$ and Danshen with $\mathrm{OB} \geq 30 \%, \mathrm{DL} \geq 0.18, \mathrm{AlgP} \leq 5$ were regarded as candidate active ingredients [28-29].

Furthermore, the target proteins corresponding to compounds were gained after clicking "Related Targets". We then converted these target names to official gene names in Uniprot KB (https://www.uniprot.org/) database with "Homo Sapiens" species.

\section{CHD-related Target Prediction}

The possible CHD-related targets were collected from three public databases including DisGeNET (https://www.disgenet.org/), NCBI Gene (https://www.ncbi.nlm.nih.gov/gene), and TTD (http://db.idrblab.net/ttd/) with the keywords "coronary heart disease". Subsequently, we aggregated these targets and removed duplicates.

\section{Common Targets Screening}

Both the ZD-related targets and CHD-related targets obtained above were imported into Venny2.1 (https://bioinfogp.cnb.csic.es/tools/venny/) to draw venny diagram, and the overlapping part showed the common targets of compounds and diseases.

\section{Protein-Protein Interaction}

Protein-Protein interaction (PPI) were performed by importing the common targets into String Whttps://string-db.org/ \database with the species restricted to "Homo Sapiens" and a highest confidence core $>0.9$ [30]. Besides, the results were exported in TSV format. 


\section{Functional Enrichment Analysis}

Gene Ontology (GO) function and Kyoto Encyclopedia of Genes and Genomes (KEGG) pathways were carried out using the clusterProfiler package in R3.6.2. To elucidate the action mechanism of ZD in the treatment of $\mathrm{CHD}$, we performed enrichment analysis on molecular functions and signaling pathways of the candidate targets with $p$ value $<0.05$ for significance, and the results were plotted as bubble chart.

\section{Network Construction and Analysis}

Cytoscape 3.7.2 was used to construct three kinds of networks including compound-target network, PPI network, and ZD-CHD network. The "degree" represents the number of edges connecting to the nodes, which is a crucial topological parameter to evaluate the significance of proteins in a visual network [31]. We considered the target with degree greater than twofold of the average as the core gene [32].

\section{Results}

\section{Compound-Target Network}

According to the screening criteria of $\mathrm{OB}, \mathrm{DL}$, and AlgP, 9 and 54 chemical components from Zhizi and Danshen were collected in the TCMSP platform. Thus, total 62 active ingredients in ZD were obtained, of which isoimperatorin is the shared ingredient of both. The characteristics of these compounds are shown in Table 1.

Next, potential targets related to the compounds were also identified from the TCMSP database. After being checked by Uniprot KB, 199 predictive targets were explored. The compound-target network of ZD were constructed using Cytoscape3.7.2, including 261 nodes and 894 edges (Figure 1).

\section{Analysis of Targets on CHD}

Based on DisGeNET, NCBI Gene, and TTD database, we collected 912, 377, and 2 targets, respectively. The detailed information of target genes were listed in Supplementary Table S1. After merging the genes from the three databases, a total of 1033 underlying action targets were identified. We then utilized the venny2.1 platform to draw a venny diagram (Figure 2), and found 83 targers were common to ZD and CHD (Supplementary Table S2). We speculate that these target genes may play an important role for ZD in the treatment of CHD.

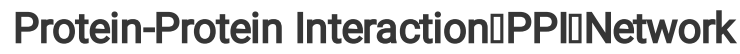

We imported the 83 common targets into the String database to acquire target proteins interactions. Subsequently, the PPI network was further visualized using Cytoscape3.7.2 by importing the results previously saved in TSV format. In addition, the network could be adjusted according to the degree which was calculated by NetworkAnalyzer tool. The smaller the degree, the smaller size and brighter color of 
node is; otherwise, the larger size and darker color of node is in the network. The PPI network is shown in Figure 3 with 74 nodes and 230 edges.

\section{Core target Screening}

The topology analysis indicated the average degree in the PPI network was 6.2. Consequently, we selected the gene with degree $>12.4$ as the core target. As shown in Table 2, the core genes in descending order were TNF (degree=27), IL6 (degree=18), AKT1 (degree=17), CXCL8 (degree=16), MAPK14 (degree=15), FOS (degree=14), VEGFA (degree=14), IL1B (degree=13), IL4 (degree=13), MAPK8 (degree=13), and RXRA (degree=13).

\section{GO and KEGG Enrichment analysis}

To further clarify the effects of ZD on CHD in molecular function and metabolic pathways, we used the cluster profiler package in $\mathrm{R}$ platform for $\mathrm{GO}$ and KEGG enrichment analysis. The results obtained were in ascending order of $\mathrm{p}$ value and met the criterion of $p<0.05$. Afterwards, we choosed the top 20 significant enrichment results for analysis. The color and size of the nodes were determined according to the value of "p.adjust" and "count".

GO functional annotation analysis showed 107 enrichment results on molecular functions. The putative targets of ZD anti-CHD mainly affected receptor ligand activity, cytokine activity, cytokine receptor binding, steroid hormone receptor activity, and peptide binding (Figure 4).

Meanwhile, there were 130 signaling pathways enriched through KEGG pathway analysis. The top 20 significant pathways were shown in Figure 5. As a result, pathways closely associated with ZD anti-CHD can be divided into three functional modules $₫$ vascular endothelial function regulation (fluid shear stress and atherosclerosis $\triangle A G E-R A G E$ signaling pathway in diabetic complications, HIF-1 signaling pathway), imflammatory effects (IL-17 signaling pathway, TNF signaling pathway $₫$ Toll-like receptor signaling pathway) \and hormone regulation (relaxin signaling pathway).

\section{ZD-CHDNetwork and Analysis}

The ZD-CHD network was established to intuitively explain the molecular mechanism of ZD against CHD, which was composed of 62 active ingredients, 83 common targets, and 20 pathways, and included 165 nodes and 695 edges (Figure 6). The visual network demonstrated that ZD may play a therapeutic role through the synergistic effect of mutiple compounds with related targets and pathways. Besides, quercetin (degree=67), kaempferol (degree=27), luteolin (degree=22), tanshinone lla (degree=18) were considered as representative compounds of ZD since they connected the most number of common target genes in the network.

\section{Discussion}


The occurrence of CHD seriously endangers human's health worldwide. As is known to us, TCM has been used to treat CHD for thousands of years. Currently, TCM advances with the times and has enriched and developed the previous theories based on macroscopic syndrome differentiation and microscopic pathology, thus forming a unique treatment system for $\mathrm{CHD}$ [33]. However, due to the complexity of the active components in TCM, the specific mechanism through which it acts on CHD remains unclear. Fortunately, the emergence of network pharmacology makes up for the deficiency. It attempts to analyse the underlying mechasims of TCM acting on diseases through the virtual screening of potential active compounds, target proteins, and signaling pathways. Hence we conducted a comprehensive study on the molecular mechanism of ZD against CHD using the strategy of network pharmacology.

In the present study, a total of 62 compounds were screened by excavating the active ingredients of ZD. As shown in Figure 6, quercetin, kaempferol, luteolin, and tanshinone lla were identified as representative compounds. Previous studies have reported that quercetin and kaempferol are flavonoids from Zhizi and have biological activities against CHD such as anti-oxidation, anti-inflammation, inhibition of platelet activation, and relaxation of blood vessels[36]. Meanwhile, luteolin is a polyphenolic compound from Danshen, which exerts cardiovascular protective effects for the roles of reducing oxidative stress, and inhibiting inflammation and apoptosis[37]. Tanshinone lla is another compound existed in Danshen that can perform anti-ischemia, anti-arrhythmia, anti-atherosclerosis, and anti-coagulation[38]. The above active ingredients provided basis for the pharmacological activities of ZD acting on CHD.

Analysis of the PPI network based on the common targets of ZD and CHD indicated that TNF, IL6, AKT1, CXCL8, MAPK14, FOS, VEGFA, IL1B, IL4, MAPK8, and RXRA were selected as the core targets. These target genes were mainly linked to the release and inhibition of inflammation and regulation of angiogenesis, which explained the effects of anti-CHD at the molecular levels. For instance, TNF is a cytokine produced by activated macrophages. Further, TNF- $a$ is an important pro-inflammatory cytokine which participates in the vasodilatation formation and mediates the recruitment of neutrophils and macrophages to sites of inflammation by stimulating endothelial cells to produce adhesion molecules[39]. IL6, an inflammatory mediator released by neutrophils, monocytes and cardiomyocytes, is associated with the thrombosis of atherosclerotic plaque. It has been reported that the elevated level of IL-6 is related to acute myocardial ischemia and serve as an indicator of recurrent CHD among patients[40]. Moreover, there are various pro-inflammatory cytokines including IL1B and CXCL8, which involved in the process of plaque formation and rupture via different pathways[41-42]. However, IL4 is an anti-inflammatory cytokine that inhibits the expression and release of inflammatory cytokines such as IL1, IL-6, and TNF-a[43-44]. Szkodzinski et al.[45] have determined prognostic implications of IL-4 in developing severe cardiac dysfunction in the course of acute myocardial infarction (AMI). In addition, AKT1 is the main isomer in vascular endothelial cells, which plays a crucial role in cardiac growth, contraction and coronary angiogenesis[46]. MAPK14, also named as p38a MAPK, is a member of p38 MAPK family. Studies have found that cardiac myocyte $p 38$ a kinase regulates angiogenesis via myocyteendothelial cell cross-talk during stress-induced remodeling in the heart[47]. VEGFA is a mitogen that promotes vascular endothelial cell proliferation and angiogenesis[48]. It has been suggested that endothelial dysfunction caused by VEGFA may increase the risk of CHD[49]. Furthermore, the 
combination of VEGFA and its receptor VEGFR2 can activate downstream signaling pathways such as MAPK, Akt to stimulate angiogenesis[50].

Through KEGG pathway enrichiment analysis, seven signaling pathways including fluid shear stress and atherosclerosis, AGE-RAGE signaling pathway in diabetic complications, HIF-1 signaling pathway, IL-17 signaling pathway, TNF signaling pathway, Toll-like receptor signaling pathway, and relaxin signaling pathway were relevant to vascular endothelial function regulation, inflammatory effects, and hormone regulation. It is worth mentioning that endothelial dysfunction plays a significant role in the progression of atherosclerotic plaque which is a main factor resulting in developing CHD. Endothelial shear stress heavily influences endothelial function, and low shear stress with the proatherogenic effect can be independent predictor of revascularization[51]. Additionally, low shear stress may drive epicardial endothelial dysfunction and atherosclerosis progression, thereby causing more advanced phenotypic manifestations of $\mathrm{CHD}$ [52]. Studies have shown that low shear stress activated the expression of vascular cell adhesion molecule 1 (VCAM-1) which was induced by cytokines such as IL-1 and TNF-a, making the markedly greater monocyte binding to the carotid[53-54]. Nevertheless, the activation of AKT1 mediated by fluid shear stress can promote cell survival in endothelial cells[55]. Advanced glycation end products (AGEs) interacting with a cell surface receptor, RAGE was reported to evoke inflammatory and thrombogenic reactions, which contributed to the development of $\mathrm{CHD}[56]$. Research found that AGE/RAGE induced the activation of the ERK/MAPK signaling pathway, ultimately leading to activate nuclear factor NF-KB, thus increasing production of proinflammatory and proatherogenic mediators [57]. Moreover, regulating the AGE/RAGE pathway may be able to treat cardiac ischemic-reperfusion injury, diabetic cardiomyopathy, and inflammatory heart diseases[58]. Hypoxia-inducible factor 1 (HIF-1) signaling pathway plays an important regulatory role in the hypoxia response. It can upregulate the expression of angiogenic factors such as vascular endothelial growth factor (VEGF) to promote angiogenesis. Besides, HIF-1 activates inducible nitric oxide to increase blood flow and reduce ischemic injury[59-60].

In addition, recent studies supported the involvement of chronic inflammation in the progression and initiation of atherosclerosis, which resulted in various cardiovascular diseases[61]. IL-17, derived by $T$ helper-17 (Th17) cells, is involved in inflammatory pathology of various diseases [62]. It promotes the release of proinflammatory cytokines (IL6, TNF-a, IL1B) and chemokines (IL8, CXCL1) to mediate inflammation. Studies found IL-17 signaling activated downstream pathways such as NF-kB and MAPK to stimulate cytokines with relevance to atherosclerosis [63]. Further, it was identified that downregulating IL-17 expression could suppress inflammation and improve heart function[64]. TNF signaling also induces inflammatory response by activating the expression of proinflammatory cytokines and transcription factors[65]. For example, TNF combined with TNF receptor 1 (TNFR1) mediated the activation of NF-kB, which was involved in the pathological process of cardiovascular disease such as ischemia-reperfusion, heart failure, and ventricular remodeling[66]. Moreover, Toll-like receptor (TLR), belongs to pattern recognition receptors, can activate expression of a series of genes related to inflammation and immune response[67]. TLRs have eleven types, of which TLR4 plays an extremely important role in CHD[68]. It was reported that TLR4 interacting with some other pathways such as 
$\mathrm{PI} 3 \mathrm{~K} / \mathrm{Akt}$ and MAPK activated a variety of downstream transcriptional factors, and initiated inflammatory response. [69].

Finally, relaxin signaling is a pathway associated with hormone regulation. Produced by the heart, relaxin coupling with its cognate receptor mediates the production of cyclic adenosine monophosphate (cAMP), and activation of nitric oxide (NO) pathway, PI3K/Akt pathway, MAPK pathway, and nuclear factor NF-KB, of which NO production plays a central role in cardioprotection[70]. Some experiments have found that relaxin is able to promote vasodilatation and angiogenesis, inhibite inflammation, and ameliorate ischemia/reperfusion injury because of the upregulated expression in ischemic heart disease [71]. Besides, in cardiac ischemia model, relaxin was verified to reduce interstitial collagen accumulation and cardiac hypertrophy, thus exerting antifibrotic effects[72].

\section{Conclusions}

To summarize, the present network pharmacology analysis revealed the potential mechanisms of ZD in the treatment of $\mathrm{CHD}$ through components and targets screening, functional enrichment analysis, and network construction. As a result, quercetin, kaempferol, luteolin, and tanshinone lla as the major active ingredients of ZD were predicted to prevent $\mathrm{CHD}$ via regulating vascular endothelial function and inflammatory effects, promoting vasodilatation, and preventing cardiac fibrosis, which acted on the key targets such as TNF, IL6, AKT1, CXCL8, MAPK14, and VEGFA. Therefore冈this study demonstrated the multi-component, multi-target and multi-pathway mechansims of ZD against $\mathrm{CHD}$, and provided a possible direction for the development of alternative therapies for CHD. In the future, we will strive to clarify our findings with basic experiments in vivo.

\section{Abbreviations}

ZD:Zhizi-Danshen; CHD: coronary heart disease; TCM: Traditional Chinese Medicine; CK: creatine kinase; LDH: lactate dehydrogenase; SOD: superoxide dismutase; MDA: decreasing malondialdehyde; TCMSP: Traditional Chinese Medicine System Pharmacology; MW: molecular weight; OB: oral bioavailability; DL: drug likeness; AlgP: oil-water partition coefficient; PPI: Protein-Protein interaction; GO: Gene Ontology; KEGG: Kyoto Encyclopedia of Genes and Genomes; VCAM-1: vascular cell adhesion molecule 1; AGEs: Advanced glycation end products; HIF-1: Hypoxia-inducible factor 1; VEGF: vascular endothelial growth factor; Th17: T helper-17; TNFR1: TNF receptor 1; TLR: Toll-like receptor; cAMP: cyclic adenosine monophosphate; NO: activation of nitric oxide.

\section{Declarations}

\section{Acknowledgements}

Not applicable.

\section{Authors' contributions}


S-YH and W-SL conceived the research methods. WN and D-YC collected and analyzed the data. ZQ and S-JP coordinated and inspected all aspects of the research design. S-YH and W-SL drafted the manuscript. All authors read and approved the final manuscript.

\section{Availability of data and materials}

The data and materials generated or analyzed during this study are available from the corresponding author on reasonable request.

\section{Ethics approval and consent to participate}

Not applicable.

\section{Consent for publication}

The manuscript is approved by all authors for publication.

\section{Competing interests}

The authors declare no conflicts of interest.

\section{Funding}

This work was supported by the National Natural Science Foundation of China (grant number: 81873274).

\section{References}

1. Khoo CM, Tai ES. Trends in the Incidence and Mortality of Coronary Heart Disease in Asian Pacific Region-The Singapore Experience. Journal of Atherosclerosis and Thrombosis. 2014; 21(Suppl 1): s2-s7.

2. Brown TM, Deng LQ, Becker DJ, et al. Trends in mortality and recurrent coronary heart disease events after an acute myocardial infarction among Medicare beneficiaries, 2001-2009. American Heart Journal. 2015;170(2):249-254.

3. Henderson A. Coronary heart disease: overview. Lancet. 1996;348(Suppl 1): s1-s4.

4. O'Rourke RA, Chatterjee K, Wei JY. Cardiovascular disease in the elderly. Coronary Heart Disease. Journal of Atherosclerosis and Thrombosis. 1987;10(2):52A-56A.

5. Puddu PE, Menotti A. Natural history of coronary heart disease and heart disease of uncertain etiology: Findings from a 50-year population study. International Journal of Cardiology. 2015;197:260-264.

6. Sagita VA, Bahtiar A, Andrajati R. Evaluation of a Clinical Pharmacist Intervention on Clinical and Drug-Related Problems Among Coronary Heart Disease Inpatients. Sultan Qaboos University Medical 
Journal. 2018;18(1): e81-e87.

7. Earnshaw SR, McDade CL, Chu YF, Fleige LE, Sievenpiper JL. Cost-effectiveness of Maintaining Daily Intake of Oat $\beta$-Glucan for Coronary Heart Disease Primary Prevention. Clinical Therapeutics. 2017; 39(4):804-814.

8. Hopkins AL. Network pharmacology. Nature Biotechnology. 2007;25(10):1110-1111.

9. Chen W, Li EC, Zhang WR. Policies on Chinese Medicine in China May Have Enlightenments to Complementary and Alternative Medicine in the World. Chinese Journal of Integrative Medicine. 2018;24(10):789-793.

10. Li DD, Huang Y, Zhang M, Lin Q, Yin HJ. Study on Anti-thrombotic Active Ingredients and Mechanisms of Radix Panacis Quinquefolii and Salviae Miltiorrhizae Based on Network Pharmacology. Chinese Journal of Integrative Medicine on Cardio-cerebrovascular Disease. 2019;17( 9):1290-1296.

11. Li C, Du X, Liu Y, et al. A Systems Pharmacology Approach for Identifying the Multiple Mechanisms of Action for the Rougui-Fuzi Herb Pair in the Treatment of Cardiocerebral Vascular Diseases. Evidence-Based Complementary and Alternative Medicine. 2020;2020:Article ID 5196302, 17 pages.

12. Li XH, Huang MX, Huang JF, et al. Interpretation of theoretical basis of therapy of activating blood, clearing heat from the nutritive level, removing toxic substances on coronary heart disease/thoracic obstruction based on TCM concept of blood stasis, heat and toxin. Journal of Beijing University of Traditional Chinese Medicine. 2017; 40(2):112-115.

13. Li ZX, Shen J, Bi W, He CN, Peng Y. Research progress of Gardenia resources and utilization in China. Journal of Chinese Medicinal Materials. 2017; 40(2):498-502.

14. Su CY, Ming QL, Khalid R, Han T, Qin LP. Salvia miltiorrhiza: Traditional medicinal uses, chemistry, and pharmacology. Chinese Journal of Natural Medicines. 2015;13(3):0163-0182.

15. Zhang HY, Wu WK, Yang JX, et al. Effect of Gardeniae Fructus on Cardiovascular System. Chinese Journal of Experimental Traditional Medical Formulae. 2011;17(14):294-296.

16. Meng XL, Li HW, Li Y, Yu Q, Wan LL, Guo C. Advances in studies on chemical constituents and pharmacological activities of Gardenia jasminoides. Chinese Journal of New Drugs. 2011;20(11):959-966.

17. Zhang ZJ, Yu W, Tan WX, et al. A Review of Danshen Combined with Clopidogrel in the Treatment of Coronary Heart Disease. Evidence-Based Complementary and Alternative Medicine. 2019;2019: Article ID 2721413, 17 pages.

18. Wan XH, Wang YL, Zhou CZ, Guo H, Ma S, Wang LZ. Research progress on chemical constituents and pharmacological effects of Salvia miltiorrhiza. Chinese Traditional and Herbal Drugs. 2020;51(3):788-795.

19. Wang T. Research Progress in Effective Constituents and Pharmacological Effects of Gardenia Jasminoides. China Pharmacist. 2015; 18(10):1782-1783.

20. Wang LL, Ma RF, Liu CY, et al. Salvia miltiorrhiza: A Potential Red Light to the Development of Cardiovascular Diseases. Current Pharmaceutical Design. 2017;23(7): 1077-1097. 
21. Hopkins AL. Network pharmacology: the next paradigm in drug discovery. Nature Chemical Biology. 2008; 4(11):682-688.

22. Li S, Zhang B. Traditional Chinese medicine network pharmacology: theory, methodology and application. Chinese Journal of Natural Medicines. 2013; 11(2):110-120.

23. Zhang RZ, Yu SJ, Bai H, Ning K. TCM-Mesh: The database and analytical system for network pharmacology analysis for TCM preparations. Scientific Reports. 2017;7: 2821.

24. Ru JL, Li P, Wang JA, et al. TCMSP: a database of systems pharmacology for drug discovery from herbal medicines. Journal of Cheminformatics. 2014; 6:13.

25. Gomez-Orellana I. Strategies to improve oral drug bioavailability. Expert Opinion on Drug Delivery. 2005;2(3):419-425.

26. Xu X, Zhang C, Li PD, et al. Drug-symptom networking: Linking drug-likeness screening to drug discovery. Pharmacological Research. 2016;103: 105-113.

27. Bao QY, Liu AY, Ma Y, et al. The effect of oil-water partition coefficient on the distribution and cellular uptake of liposome-encapsulated gold nanoparticles. Colloids and Surfaces B: Biointerfaces. 2016; 146:475-481.

28. Chen H, Gao X, Zhao W, et al. Mechanism of Gardenia jasminoides against cholestasis based on network pharmacology. Zhongguo Zhong Yao Za Zhi. 2019;44(13):2709-2718.

29. Yang WN, Xu WH, Xu XM, Chen X, Zheng JH. Mechanism \esearch of \adix Salviae-Radix Paeoniae Rubra on Treating Acute Myocardial Infarction Based on Network Pharmacology. Liaoning Journal of traditional Chinese Medicine. 2020; 47(1):141-145.

30. Gong BJ, Kao YL, Zhang CG, Zhao HS, Sun FD, Gong ZH. Exploring the Pharmacological Mechanism of the Herb Pair 'HuangLian-GanJiang' against Colorectal Cancer Based on Network Pharmacology. Evidence-Based Complementary and Alternative Medicine. 2019;2019:Article ID 2735050, 12 pages.

31. Ma Y, Wang WJ, Wang SJ, et al. Study on the mechanism of Danshen-Gegen in treating coronary heart disease based on network pharmacology. Natural Product Research and Development. 2020; http://kns.cnki.net/kcms/detail/51.1335.q.20200107.1417.002.html, 21 pages.

32. He XY, Zheng SC, Wang Q, Wu JM, Chen WN, Zhang Z. Potential molecular mechanisms of Huanglian Jiedu Decoction in treatment of atherosclerosis based on network pharmacology. Chinese Traditional and Herbal Drugs. 2020; http://kns.cnki.net/kcms/detail/12.1108.R.20200122.1128.004.html, 10 pages.

33. Xu JX, Wu H, Chu QM, Zhou XX. Primary Study on the Pathogenesis of Coronary Heart Disease with Toxic Stasis. Journal of Liaoning University of TCM. 2019;21(11):170-173.

34. Tang XL, Liu JX, Li P, et al. Protective effects of kaempferol and quercetin on hypoxia/reoxygenation and peroxidation injury in neonatal cardiomyocytes. Pharmacology and Clinics of Chinese Materia Medica. 2012; 28(1):56-58.

35. Nai CS, Xuan HC, Zhang YY, et al. Luteolin Exerts Cardioprotective Effects through Improving Sarcoplasmic Reticulum $\mathrm{Ca}^{2+}$-ATPase Activity in Rats during Ischemia/Reperfusion In Vivo. 
Evidence-Based Complementary and Alternative Medicine. 2015;2015,:Article ID 365854, 12 pages.

36. Qin WW, Wang L, Jiao Z, et al. Lower clearance of sodium tanshinone IIA sulfonate in coronary heart disease patients and the effect of total bilirubin: a population pharmacokinetics analysis. Chinese Journal of Natural Medicines. 2019; 17(3):0218-0226.

37. Jing X, Chen SS, Jing W, Tan Q, Yu MX, Tu JC. Diagnostic Potential of Differentially Expressed Homer1, IL-1 $\beta$, and TNF-a in Coronary Artery Disease. International Journal of Molecular Sciences. 2015;15:535-546.

38. Zhao L, Wang XL, Yang YH. Association between interleukin- 6 and the risk of cardiac events measured by coronary computed tomography angiography. International Journal of Cardiovascular Imaging. 2017; 33(8):1237-1244.

39. Rai H, Sinha N, Kumar S, Sharma AK, Agrawal S. Interleukin-1 Gene Cluster Polymorphisms and Their Association with Coronary Artery Disease: Separate Evidences from the Largest Case-Control Study amongst North Indians and an Updated Meta-Analysis. PLoS One. 2016;11(4): Article ID e0153480.

40. Inoue T, Komoda H, Nonaka M, Kameda M, Uchida T, Node K. Interleukin-8 as an independent predictor of long-term clinical outcome in patients with coronary artery disease. International Journal of Cardiology. 2008; 124:319-325.

41. Sobti RC, Maithil N, Thakur H, Sharma Y, Talwar KK. VEGF and IL-4 gene variability and its association with the risk of coronary heart disease in north Indian population. Molecular and Cellular Biochemistry. 2010; 341:139-148.

42. Basol N, Celik A, Karakus N, Ozsoy SD, Yigit S. The Evaluation of Angiotensin-converting Enzyme (ACE) Gene I/D and IL-4 Gene Intron 3 VNTR Polymorphisms in Coronary Artery Disease. In Vivo. $2014 ; 28(5): 983-988$.

43. Szkodzinski J, Hudzik B, Osuch M, et al. Serum concentrations of interleukin-4 and interferon-gamma in relation to severe left ventricular dysfunction in patients with acute myocardial infarction undergoing percutaneous coronary intervention. Heart Vessels. 2011;26(4): 399-407.

44. Vandoorne K, Vandsburge MH, Raz T, et al. Chronic Akt1 Deficiency Attenuates Adverse Remodeling and Enhances Angiogenesis After Myocardial Infarction. Circulation-Cardiovascular Imaging. 2013; 6(6):992-1000.

45. Rose BA, Yokota T, Chintalgattu V, et al. Cardiac Myocyte p38\ Kinase Regulates Angiogenesis via Myocyte-Endothelial Cell Cross-talk During Stress-Induced Remodeling in the Heart. The Journal of Biological Chemistry. 2013;6(6):992-1000.

46. Ma YH, Zechariah A, Y. Qu, and D. M. Hermann. Effects of Vascular Endothelial Growth Factor in Ischemic Stroke. Journal of Neuroscience Research. 2012;90(10):1873-1882.

47. Liu DX, Song JT, Ji XF, Liu ZQ, Cong ML, Hu B. Association of Genetic Polymorphisms on VEGFA and VEGFR2 With Risk of Coronary Heart Disease. Medicine. 2016;95(19):Article ID e3413.

48. Wang YB, Zheng Y, Zhang WL, et al. Polymorphisms of KDR Gene Are Associated With Coronary Heart Disease. Journal of the American College of Cardiology. 2007; 50(8):760-767. 
49. Yamamoto E, Siasos G, Zaromytidou M, et al. Low Endothelial Shear Stress Predicts Evolution to High-Risk Coronary Plaque Phenotype in the Future: A Serial Optical Coherence Tomography and Computational Fluid Dynamics Study. Circulation Cardiovascular Interventions. 2017;10(8): Article ID e005455.

50. Vergallo R, Papafaklis MI, Yonetsu T, et al. Endothelial Shear Stress and Coronary Plaque Characteristics in Humans: Combined Frequency-Domain Optical Coherence Tomography and Computational Fluid Dynamics Study. Circulation-Cardiovascular Imaging. 2014; 7(6):905-911.

51. DeVerse JS, Sandhu AS, Mendoza N, et al. Shear stress modulates VCAM-1 expression in response to TNF-a and dietary lipids via interferon regulatory factor-1 in cultured endothelium. American Journal of Physiology-Heart and Circulatory Physiology. 2013;305(8):H1149-H1157.

52. Surapisitchat J, Hoefen RJ, Pi XC, Yoshizumi M, Yan C, Berk BC. Fluid shear stress inhibits TNF-a activation of JNK but not ERK1y2 or p38 in human umbilical vein endothelial cells: Inhibitory crosstalk among MAPK family members. PNAS. 2001;98(11): 6476-6481.

53. Melchior B, Frangos JA. Distinctive Subcellular Akt-1 Responses to Shear Stress in Endothelial Cells. Journal of Cellular Biochemistry. 2014; 115(1): 121-129.

54. Yamagishi S, Matsui T, Ishibashi Y, et al. Phytochemicals Against Advanced Glycation End Products (AGEs) and the Receptor System. Current Pharmaceutical Design. 2017; 23(8): 1135-1141.

55. Kosmopoulos M, Drekolia D, Zavras PD, Piperi C, Papavassiliou AG. Impact of advanced glycation end products (AGEs) signaling in coronary artery disease. BBA-Molecular Basis of Disease. 2018;1865:611-619.

56. Lee TW, Kao YH, Chen YJ, Chao TF, Lee TI. Therapeutic potential of vitamin D in AGE/RAGE-related cardiovascular diseases. Cellular and Molecular Life Sciences. 2019;76(20): 4103-4115.

57. Sousa Fialho MdL, Abd Jamil AH, Stannard GA, Heather LC. Hypoxia-inducible factor 1 signalling, metabolism and its therapeutic potential in cardiovascular disease. BBA-Molecular Basis of Disease. 2019;1865:831-843.

58. Semenza GL. Hypoxia-Inducible Factor 1 and Cardiovascular Disease. Annual review of physiology. 2014;76:39-56.

59. Hansson GK. Inflammation, Atherosclerosis, and Coronary Artery Disease. The New England Journal of Medicine. 2005; 352(16):1685-1695.

60. Taleb S, Tedgui A, Mallat Z. IL-17 and Th17 Cells in Atherosclerosis Subtle and Contextual Roles. Arteriosclerosis. Thrombosis, and Vascular Biology. 2015;35(2): 258-264.

61. Chang SL, Hsiao YW, Tsai YN, et al. Interleukin-17 enhances cardiac ventricular remodeling via activating MAPK pathway in ischemic heart failure. Journal of Molecular and Cellular Cardiology. 2018;122: 69-79.

62. Chen MX, Yu LL, Liu QM, Jiang H, Zhou SH. Interleukin-17 inhibition: An important target for attenuating myocardial ischemia and reperfusion injury. International Journal of Cardiology. 2015; 198:89-90. 
63. Wallach D. The Tumor Necrosis Factor Family: Family Conventions and Private Idiosyncrasies. Cold Spring Harbor Perspectives in Biology. 2018; 10:Article ID a028431.

64. Chen GQ, Goeddel DV. TNF-R1 Signaling: A Beautiful Pathway. Science. 2002; 296:1634-1635.

65. Parizadeh SM, Ghandehari M, Heydari-majd M, et al. Toll-like Receptors Signaling Pathways as a Potential Therapeutic Target in Cardiovascular Disease. Current Pharmaceutical Design. 2018 24(17):1-12.

66. Yang Y, LV J, Jiang S, et al. The emerging role of Toll-like receptor 4 in myocardial inflammation. Cell Death and Disease. 2016; 7:Article ID e2234.

67. Fang Y, Hu JG. Toll-like receptor and its roles in myocardial ischemic/reperfusion injury. Medical Science Monitor. 2011; 17(4): RA100-RA109.

68. Valle Raleigh JM, Toldo S, Das A, Abbate A, Salloum FN. Relaxin' the Heart: A Novel Therapeutic Modality. Journal of Cardiovascular Pharmacology and Therapeutics. 2016; 21(4): 353-362.

69. Samuel CS, Du XJ, Bathgate RAD, Summers RJ.'Relaxin' the stiffened heart and arteries: The therapeutic potential for relaxin in the treatment of cardiovascular disease. Pharmacology and Therapeutics. 2006; 112(2):529-552.

70. Zhou X, Chen X, Cai JJ, et al. Relaxin inhibits cardiac fibrosis and endothelial-mesenchymal transition via the notch pathway," Drug Design, Development and Therapy. 2015;9:4599-4611.

\section{Tables}

TABLE 1: Characteristics of active ingredients in ZD 


\begin{tabular}{|c|c|c|c|c|c|c|}
\hline Molecular Name & Mol ID & Source & MW & AlgP & 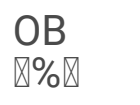 & DL \\
\hline crocetin & MOL001406 & Zhizi & 328.44 & 4.58 & 35.30 & 0.26 \\
\hline ammidin & MOL001941 & Zhizi & 270.30 & 3.65 & 34.55 & 0.22 \\
\hline geniposide & MOL004557 & Zhizi & 388.41 & -2.25 & 14.64 & 0.44 \\
\hline quercetin & MOL000098 & Zhizi & 302.25 & 1.50 & 46.43 & 0.28 \\
\hline kaempferol & MOL000422 & Zhizi & 286.25 & 1.77 & 41.88 & 0.24 \\
\hline \multirow[t]{2}{*}{ isoimperatorin } & MOL001942 & Zhizi & 270.30 & 3.65 & 45.46 & 0.23 \\
\hline & & Danshen & & & & \\
\hline chrysin & MOL002560 & Zhizi & 254.25 & 2.60 & 22.61 & 0.18 \\
\hline $\begin{array}{l}\text { 5-hydroxy-7-methoxy-2-(3,4,5- } \\
\text { trimethoxyphenyl)chromone }\end{array}$ & MOL003095 & Zhizi & 358.37 & 2.80 & 51.96 & 0.41 \\
\hline 3-methylkempferol & MOL007245 & Zhizi & 300.28 & 1.84 & 60.16 & 0.26 \\
\hline 1,2,5,6-tetrahydrotanshinone & MOL001601 & Danshen & 280.34 & 2.98 & 38.75 & 0.36 \\
\hline sugiol & MOL002222 & Danshen & 300.48 & 4.99 & 36.11 & 0.28 \\
\hline dehydrotanshinone lla & MOL002651 & Danshen & 292.35 & 4.22 & 43.76 & 0.40 \\
\hline digallate & MOL000569 & Danshen & 322.24 & 1.53 & 61.85 & 0.26 \\
\hline luteolin & MOL000006 & Danshen & 286.25 & 2.07 & 36.16 & 0.25 \\
\hline $\begin{array}{l}\text { 5,6-dihydroxy-7-isopropyl-1,1- } \\
\text { dimethyl-2,3-dihydrophenanthren-4- } \\
\text { one }\end{array}$ & MOL007036 & Danshen & 298.41 & 4.38 & 33.77 & 0.29 \\
\hline $\begin{array}{l}\text { 2-isopropyl-8-methylphenanthrene- } \\
\text { 3,4-dione }\end{array}$ & MOL007041 & Danshen & 264.34 & 4.16 & 40.86 & 0.23 \\
\hline 3a-hydroxytanshinone lla & MOL007045 & Danshen & 310.37 & 3.56 & 44.93 & 0.44 \\
\hline $\begin{array}{l}\text { (E)-3-[2-(3,4-dihydroxyphenyl)-7- } \\
\text { hydroxy-benzofuran-4-yl]acrylic acid }\end{array}$ & MOL007048 & Danshen & 312.29 & 3.21 & 48.24 & 0.31 \\
\hline 4-methylenemiltirone & MOL007049 & Danshen & 266.36 & 4.33 & 34.35 & 0.23 \\
\hline
\end{tabular}

TABLE1: Continued. 


\begin{tabular}{|c|c|c|c|c|c|c|}
\hline Molecular Name & Mol ID & Source & MW & AlgP & $\mathrm{OB} \otimes \% \rrbracket$ & DL \\
\hline $\begin{array}{l}\text { 2-(4-hydroxy-3-methoxyphenyl)-5-(3- } \\
\text { hydroxypropyl)-7-methoxy-3- } \\
\text { benzofurancarboxaldehyde }\end{array}$ & MOL007050 & Danshen & 356.40 & 3.58 & 62.78 & 0.40 \\
\hline formyltanshinone & MOL007058 & Danshen & 290.28 & 3.36 & 73.44 & 0.42 \\
\hline $\begin{array}{l}\text { 3-beta- } \\
\text { Hydroxymethyllenetanshiquinone }\end{array}$ & MOL007059 & Danshen & 294.32 & 3.16 & 32.16 & 0.41 \\
\hline methylenetanshinquinone & MOL007061 & Danshen & 278.32 & 4.26 & 37.07 & 0.36 \\
\hline przewalskin a & MOL007063 & Danshen & 398.49 & 2.25 & 37.11 & 0.65 \\
\hline przewalskin b & MOL007064 & Danshen & 330.46 & 3.18 & 110.32 & 0.44 \\
\hline przewaquinone $b$ & MOL007068 & Danshen & 292.30 & 2.99 & 62.24 & 0.41 \\
\hline przewaquinone $\mathrm{c}$ & MOL007069 & Danshen & 296.34 & 3.31 & 55.74 & 0.40 \\
\hline $\begin{array}{l}\text { (6S,7R)-6,7-dihydroxy-1,6-dimethyl- } \\
\text { 8,9-dihydro-7H-naphtho[8,7- } \\
\text { g]benzofuran-10,11-dione }\end{array}$ & MOL007070 & Danshen & 312.34 & 2.34 & 41.31 & 0.45 \\
\hline przewaquinone $f$ & MOL007071 & Danshen & 312.34 & 2.07 & 40.31 & 0.46 \\
\hline sclareol & MOL007077 & Danshen & 308.56 & 4.27 & 43.67 & 0.21 \\
\hline tanshinaldehyde & MOL007079 & Danshen & 308.35 & 3.83 & 52.47 & 0.45 \\
\hline danshenol b & MOL007081 & Danshen & 354.48 & 2.59 & 57.95 & 0.56 \\
\hline danshenol a & MOL007082 & Danshen & 336.41 & 2.01 & 56.97 & 0.52 \\
\hline salvilenone & MOL007085 & Danshen & 292.40 & 4.26 & 30.38 & 0.38 \\
\hline cryptotanshinone & MOL007088 & Danshen & 296.39 & 3.44 & 52.34 & 0.40 \\
\hline dan-shexinkum d & MOL007093 & Danshen & 336.41 & 2.83 & 38.88 & 0.55 \\
\hline danshenspiroketallactone & MOL007094 & Danshen & 282.36 & 3.24 & 50.43 & 0.31 \\
\hline
\end{tabular}

TABLE1: Continued. 


\begin{tabular}{|c|c|c|c|c|c|c|}
\hline Molecular Name & Mol ID & Source & MW & AlgP & $\mathrm{OB} \otimes \% \rrbracket$ & DL \\
\hline deoxyneocryptotanshinone & MOL007098 & Danshen & 298.41 & 4.32 & 49.40 & 0.29 \\
\hline dihydrotanshinlactone & MOL007100 & Danshen & 266.31 & 2.77 & 38.68 & 0.32 \\
\hline dihydrotanshinone I & MOL007101 & Danshen & 278.32 & 2.86 & 45.04 & 0.36 \\
\hline epidanshenspiroketallactone & MOL007105 & Danshen & 284.38 & 2.37 & 68.27 & 0.31 \\
\hline isocryptotanshi-none & MOL007108 & Danshen & 296.39 & 3.59 & 54.98 & 0.39 \\
\hline isotanshinone II & MOL007111 & Danshen & 294.37 & 4.66 & 49.92 & 0.40 \\
\hline miltionone I & MOL007119 & Danshen & 312.39 & 3.33 & 49.68 & 0.32 \\
\hline miltionone II & MOL007120 & Danshen & 312.39 & 2.14 & 71.03 & 0.44 \\
\hline miltipolone & MOL007121 & Danshen & 300.43 & 2.74 & 36.56 & 0.37 \\
\hline miltirone & MOL007122 & Danshen & 282.41 & 4.73 & 38.76 & 0.25 \\
\hline neocryptotanshinone II & MOL007124 & Danshen & 270.35 & 3.61 & 39.46 & 0.23 \\
\hline neocryptotanshinone & MOL007125 & Danshen & 314.41 & 3.01 & 52.49 & 0.32 \\
\hline $\begin{array}{l}\text { 1-methyl-8,9-dihydro-7H-naphtho[5,6- } \\
\text { g]benzofuran-6,10,11-trione }\end{array}$ & MOL007127 & Danshen & 280.29 & 3.21 & 34.72 & 0.37 \\
\hline prolithospermic acid & MOL007130 & Danshen & 314.31 & 2.77 & 64.37 & 0.31 \\
\hline $\begin{array}{l}\text { (2R)-3-(3,4-dihydroxyphenyl)-2-[(Z)-3- } \\
\text { (3,4-dihydroxyphenyl)acryloyl]oxy- } \\
\text { propionic acid }\end{array}$ & MOL007132 & Danshen & 360.34 & 2.69 & 109.38 & 0.35 \\
\hline salvianolic acid g & MOL007141 & Danshen & 340.30 & 2.20 & 45.56 & 0.61 \\
\hline salvianolic acid $\mathrm{j}$ & MOL007142 & Danshen & 538.49 & 3.78 & 43.38 & 0.72 \\
\hline salvilenone I & MOL007143 & Danshen & 270.40 & 2.88 & 32.43 & 0.23 \\
\hline salviolone & MOL007145 & Danshen & 268.38 & 4.05 & 31.72 & 0.24 \\
\hline
\end{tabular}

TABLE1: Continued. 


\begin{tabular}{|c|c|c|c|c|c|c|}
\hline Molecular Name & Mol ID & Source & MW & AlgP & 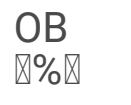 & DL \\
\hline $\begin{array}{l}\text { (6S)-6-hydroxy-1-methyl-6-methylol- } \\
\text { 8,9-dihydro-7H-naphtho[8,7- } \\
\text { g]benzofuran-10,11-quinone }\end{array}$ & MOL007150 & Danshen & 312.34 & 2.42 & 75.39 & 0.46 \\
\hline tanshindiol b & MOL007151 & Danshen & 312.34 & 2.34 & 42.67 & 0.45 \\
\hline przewaquinone e & MOL007152 & Danshen & 312.34 & 2.34 & 42.85 & 0.45 \\
\hline tanshinone lla & MOL007154 & Danshen & 294.37 & 4.66 & 49.89 & 0.40 \\
\hline $\begin{array}{l}\text { (6S)-6-(hydroxymethyl)-1,6-dimethyl- } \\
\text { 8,9-dihydro-7H-naphtho[8,7- } \\
\text { g]benzofuran-10,11-dione }\end{array}$ & MOL007155 & Danshen & 310.37 & 3.57 & 65.26 & 0.45 \\
\hline tanshinone VI & MOL007156 & Danshen & 296.34 & 2.44 & 45.64 & 0.30 \\
\hline
\end{tabular}

TABLE 2: The 11 core targets and topological parameters of PPI network

\begin{tabular}{|lllll|}
\hline Number & Target name & Degree & Betweenness Centrality & Closeness Centrality \\
\hline 1 & TNF & 27 & 0.25 & 0.54 \\
\hline 2 & IL6 & 18 & 0.08 & 0.47 \\
\hline 4 & AKT1 & 17 & 0.13 & 0.46 \\
\hline 5 & CXCL8 & 16 & 0.11 & 0.46 \\
\hline 6 & MAPK14 & 15 & 0.07 & 0.46 \\
\hline 7 & FOS & 14 & 0.08 & 0.44 \\
\hline 8 & IL1B & 13 & 0.01 & 0.47 \\
\hline 9 & IL4 & 13 & 0.06 & 0.43 \\
\hline 10 & MAPK8 & 13 & 0.07 & 0.43 \\
\hline 11 & RXRA & 13 & 0.16 & 0.45 \\
\hline
\end{tabular}

\section{Figures}




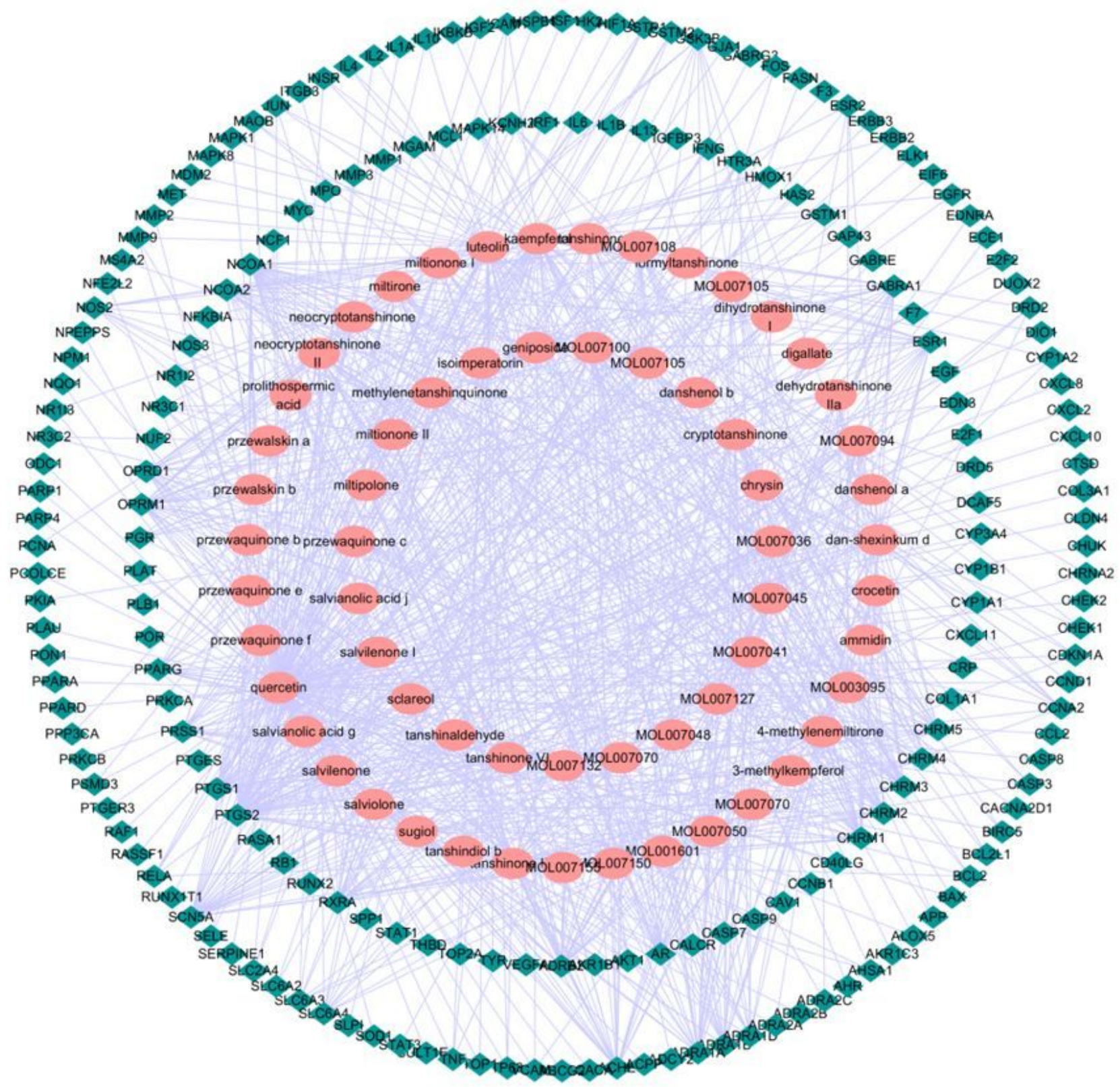

Figure 1

Compound-target network of ZD. Green diamond represents the predictive target proteins, while pink ellipse represents the potential active ingredients of ZD. 




Figure 1

Compound-target network of ZD. Green diamond represents the predictive target proteins, while pink ellipse represents the potential active ingredients of ZD. 


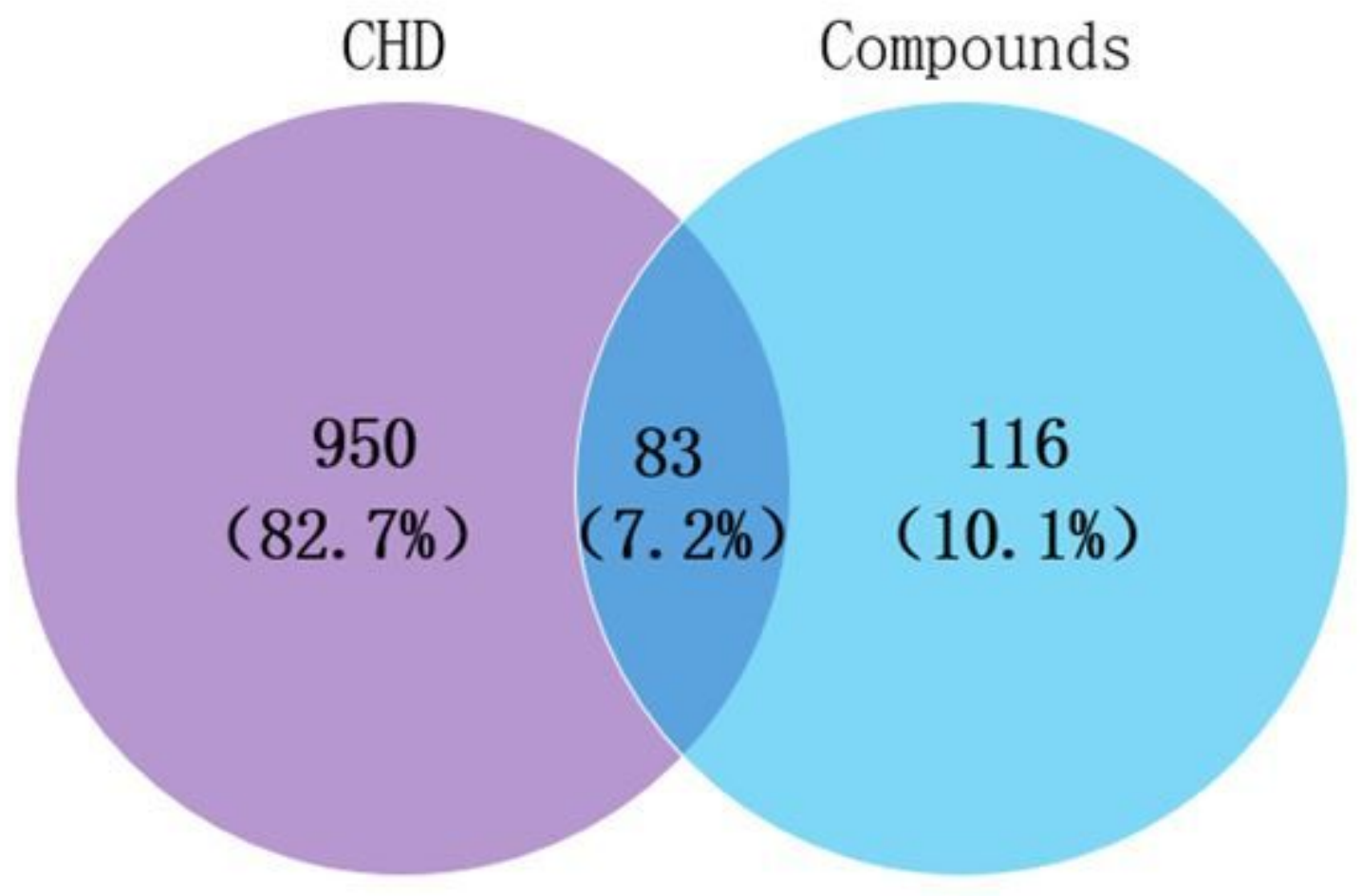

Figure 2

Venny diagram of 83 common targets in compounds and $\mathrm{CHD}$. The purple represents the target genes related to $\mathrm{CHD}$, while the blue reprents the target genes related to compounds in $\mathrm{ZD}$.

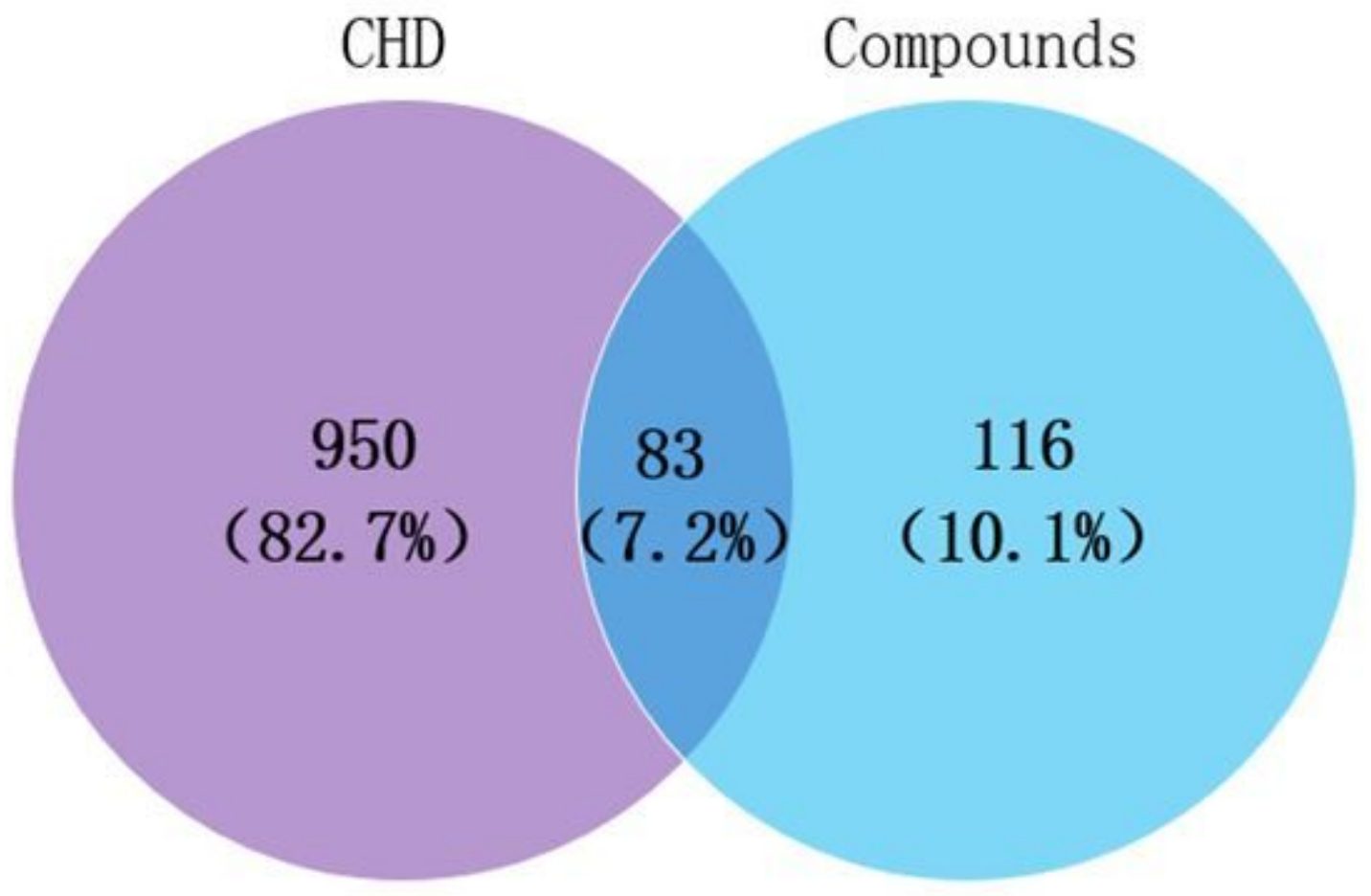

Figure 2 
Venny diagram of 83 common targets in compounds and CHD. The purple represents the target genes related to $\mathrm{CHD}$, while the blue reprents the target genes related to compounds in ZD.

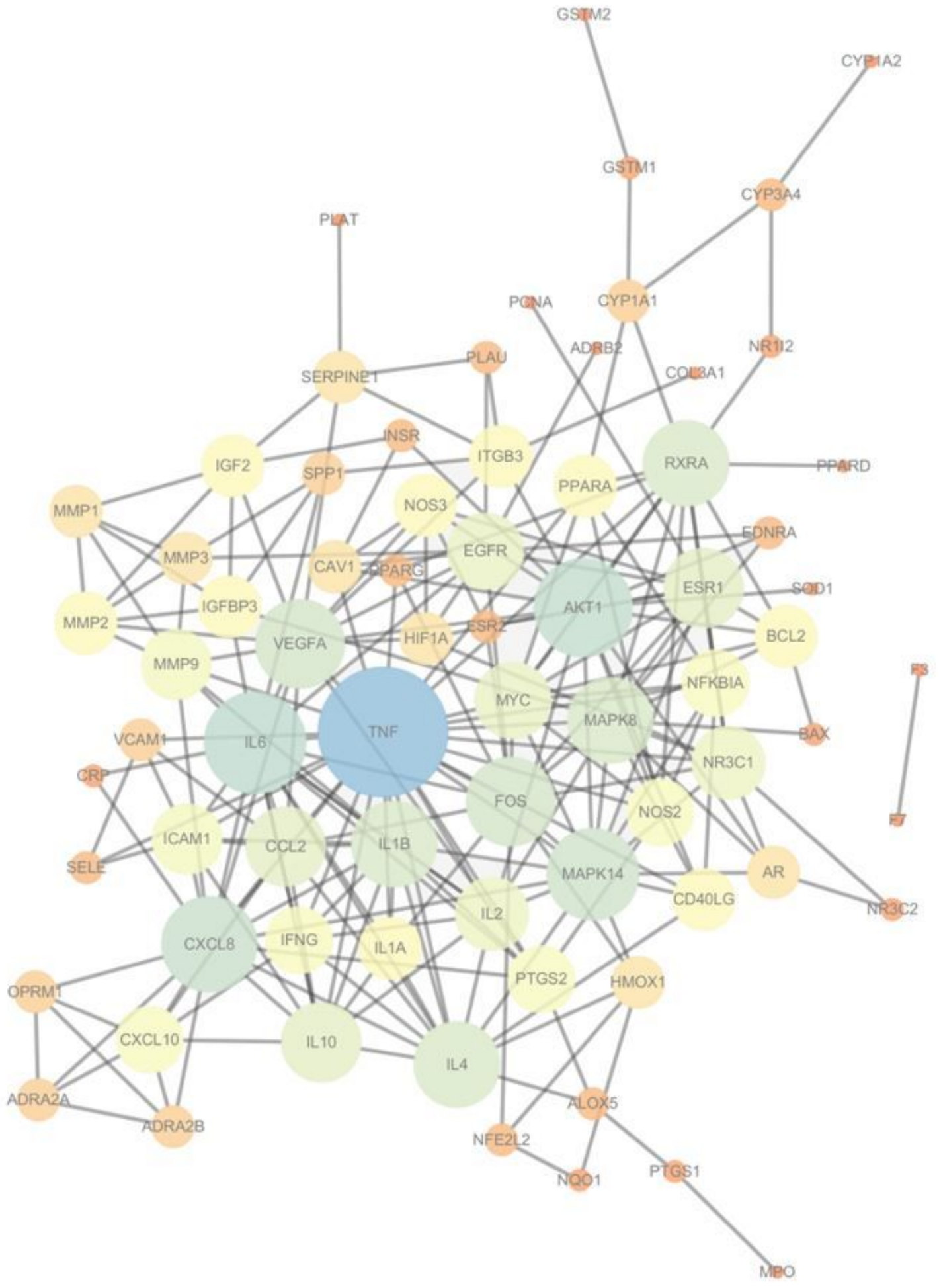

Figure 3

Protein-protein interaction (PPI) network of common targets represents the reaction of ZD response to $\mathrm{CHD}$. Different sizes and colors of the nodes represent the degree. 


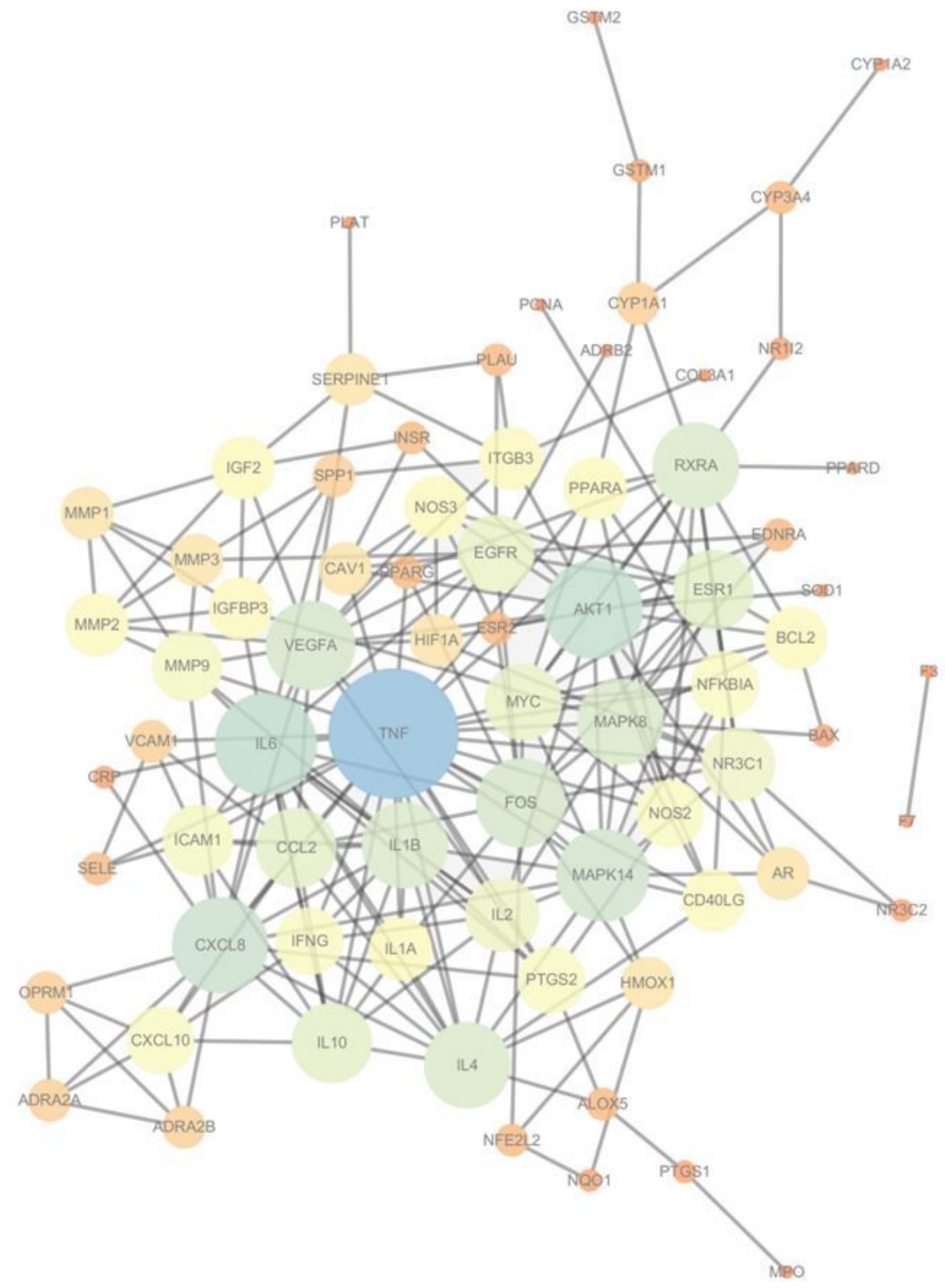

\section{Figure 3}

Protein-protein interaction (PPI) network of common targets represents the reaction of ZD response to $\mathrm{CHD}$. Different sizes and colors of the nodes represent the degree. 


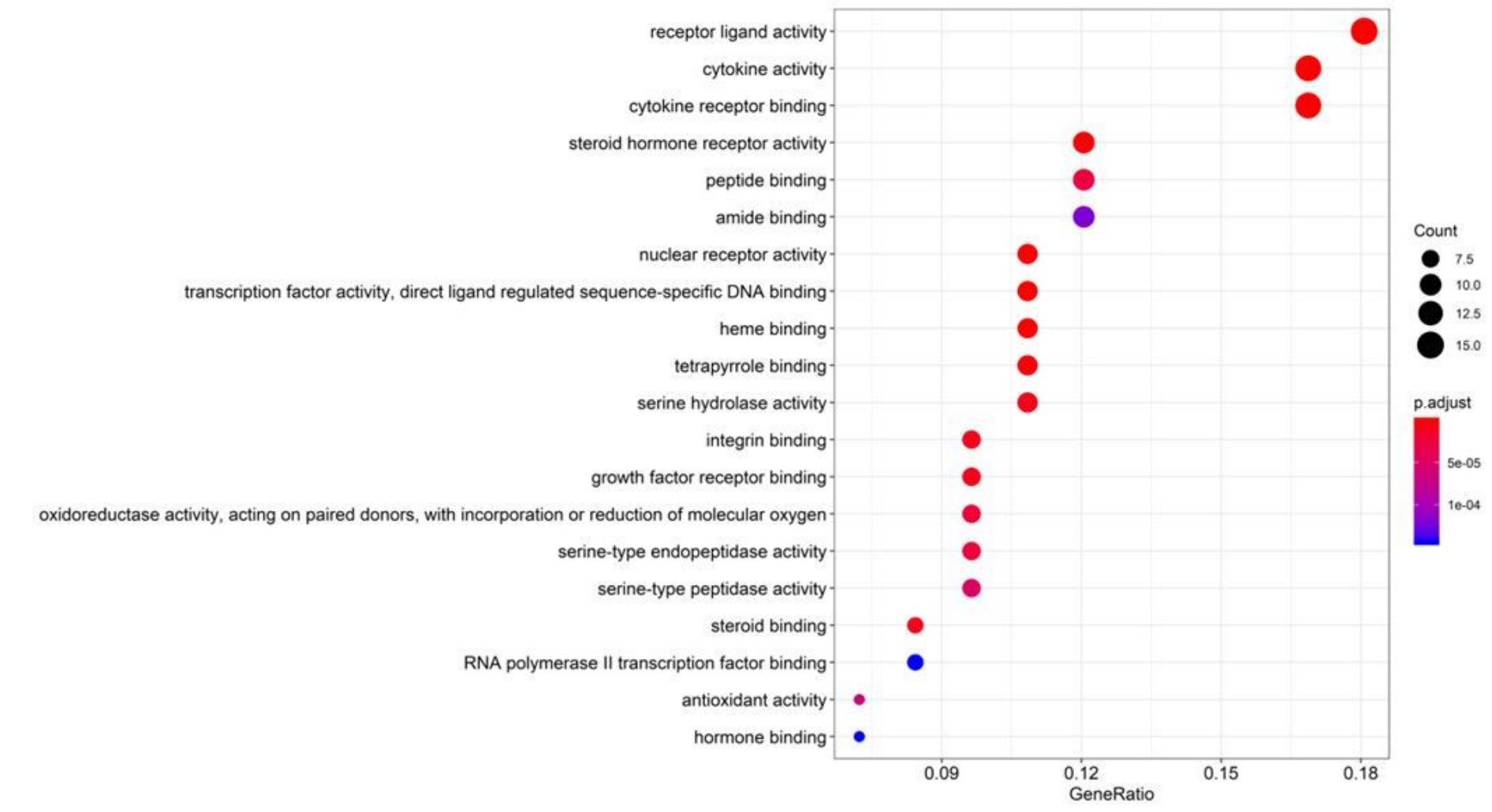

\section{Figure 4}

\section{Top 20 enriched results of GO analysis for molecular function.}

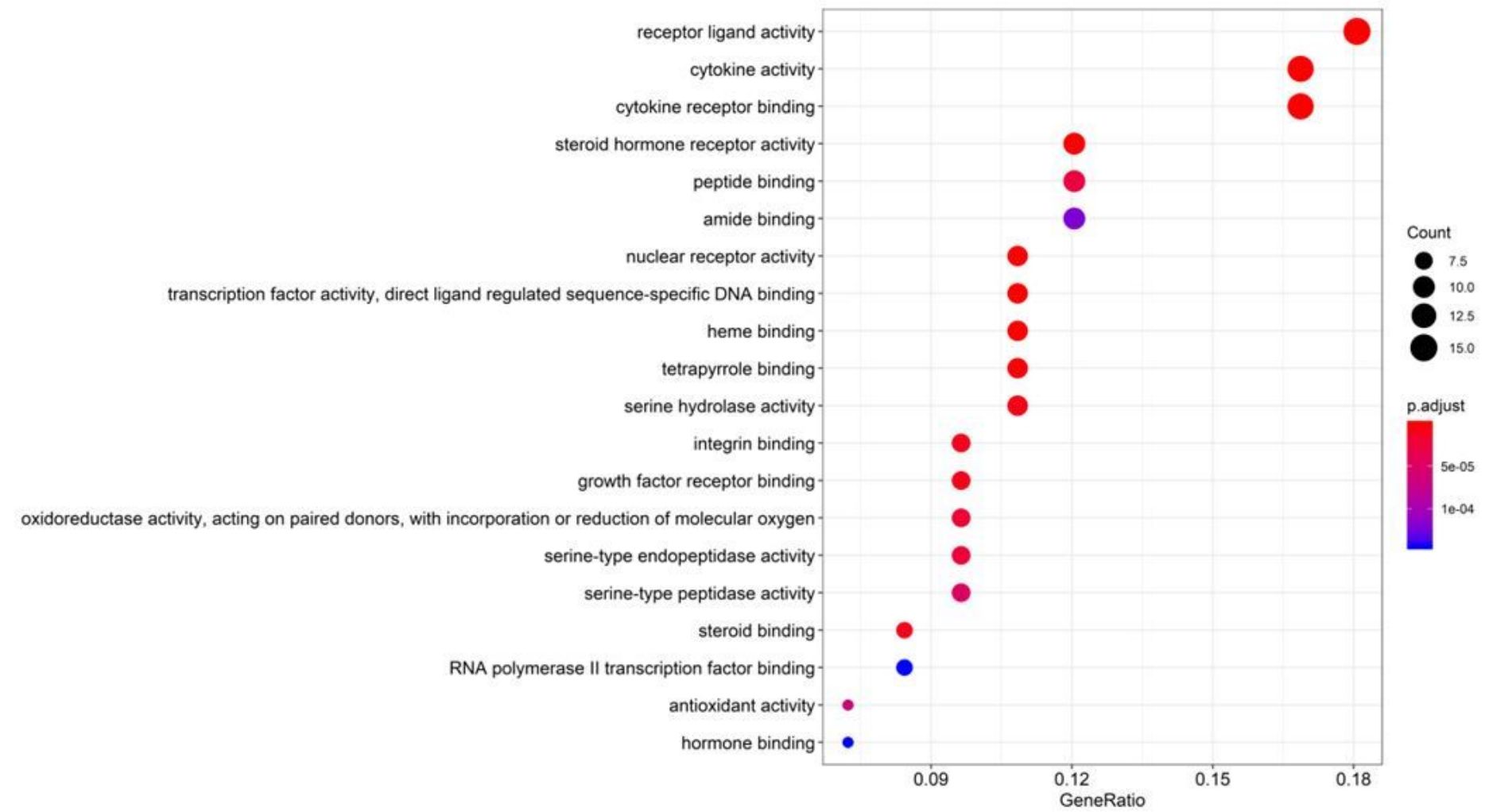

Figure 4 
Top 20 enriched results of GO analysis for molecular function.

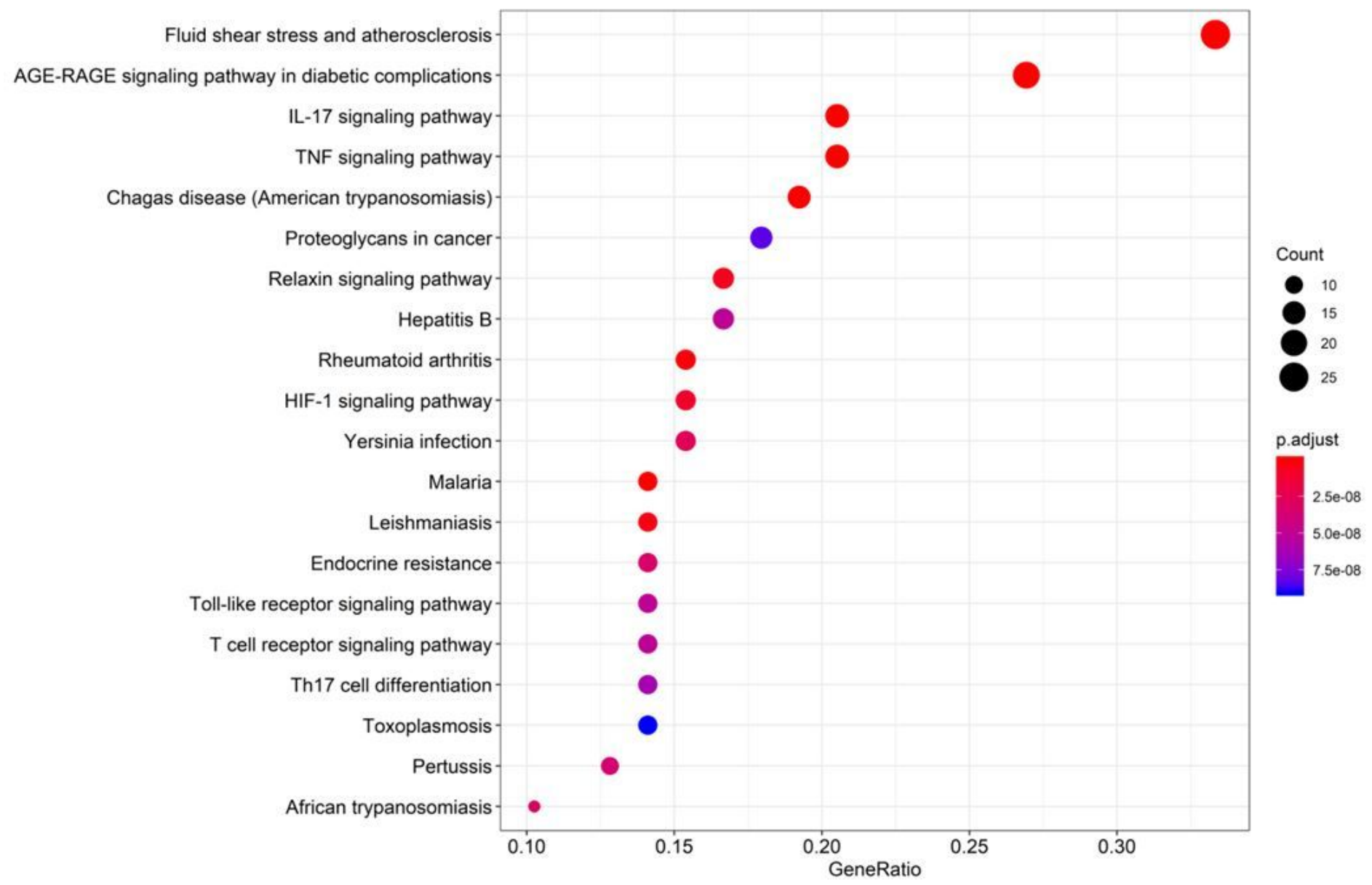

\section{Figure 5}

Top 20 enriched results of KEGG pathways analysis. 


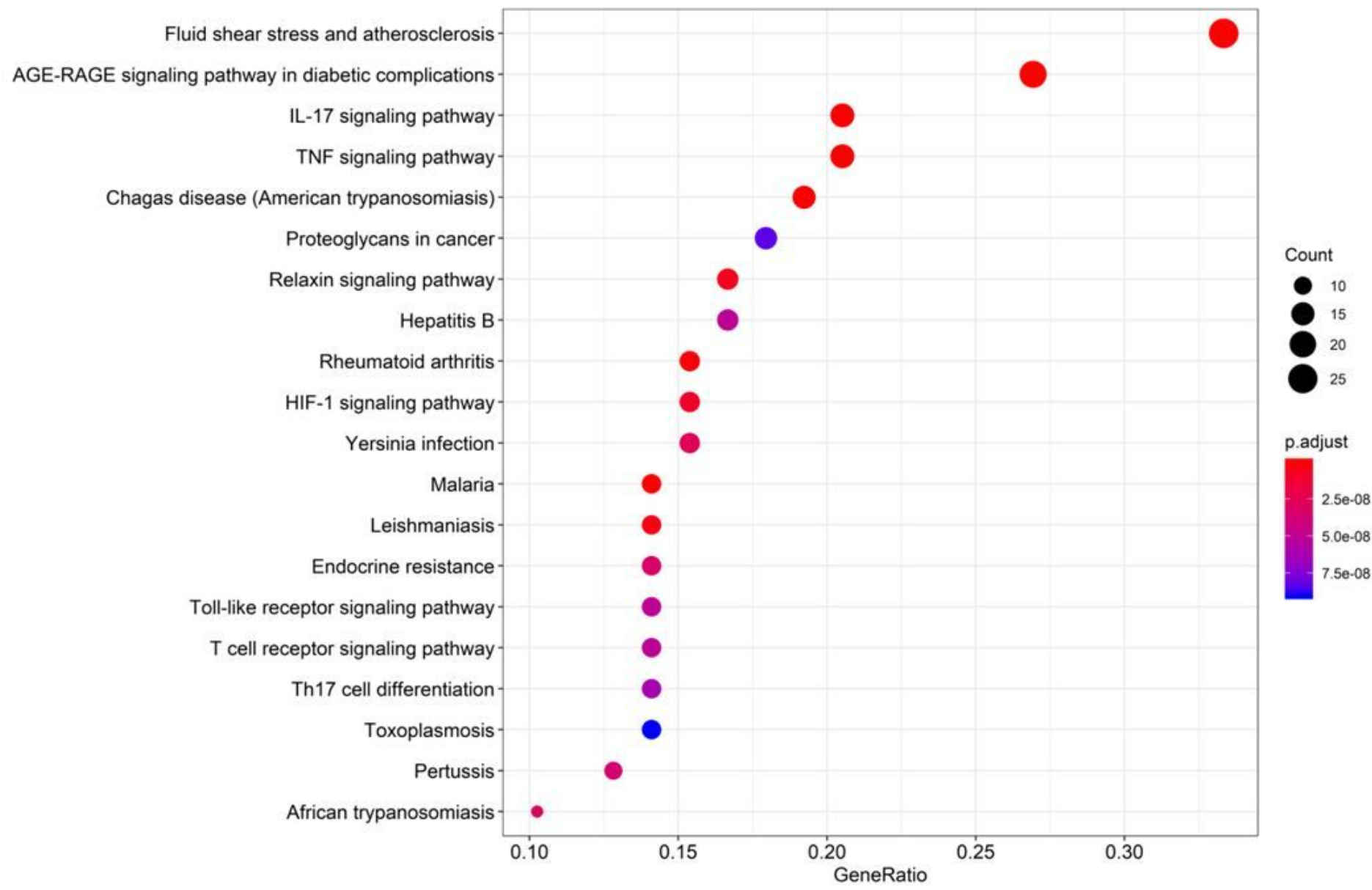

Figure 5

Top 20 enriched results of KEGG pathways analysis.

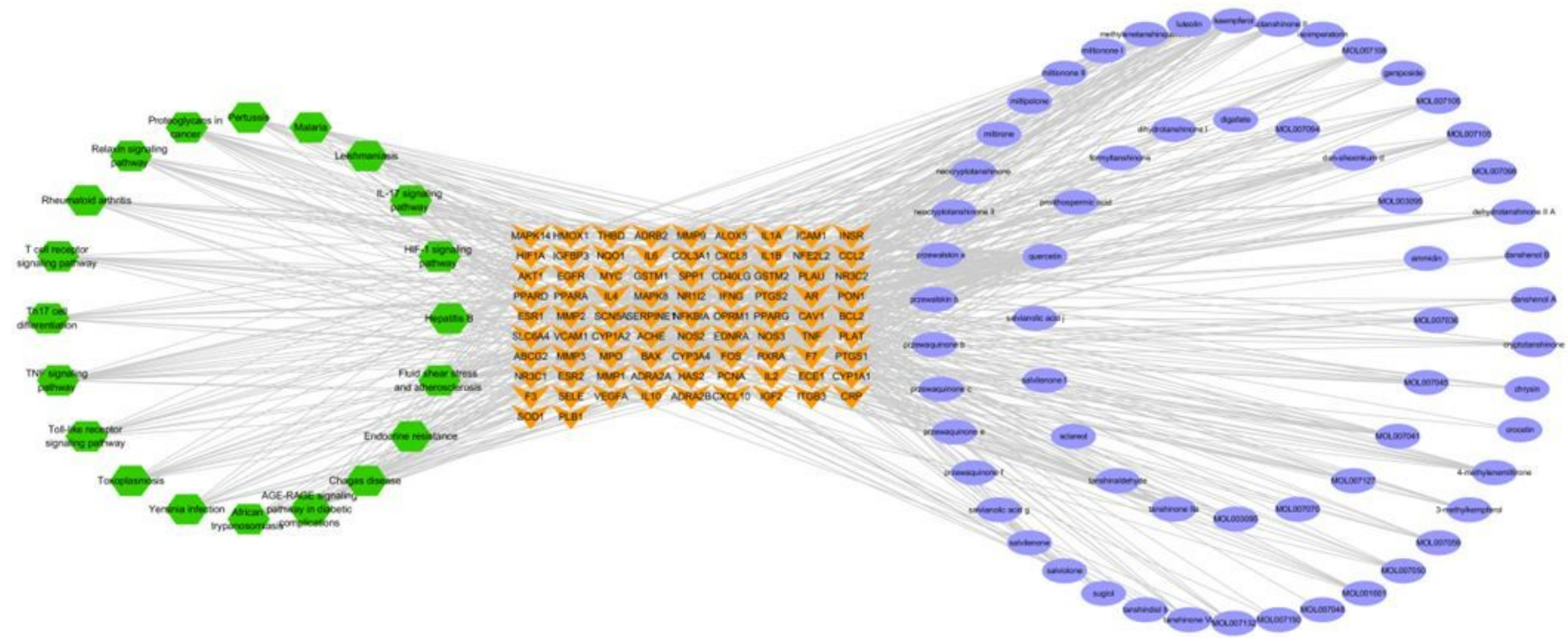

Figure 6 
ZD-CHD network. Purple ellipse represents the putative therapeutic active ingredients of ZD, orange arrow represents the common targets from ZD and CHD, and green hexagon represents the KEGG pathways.

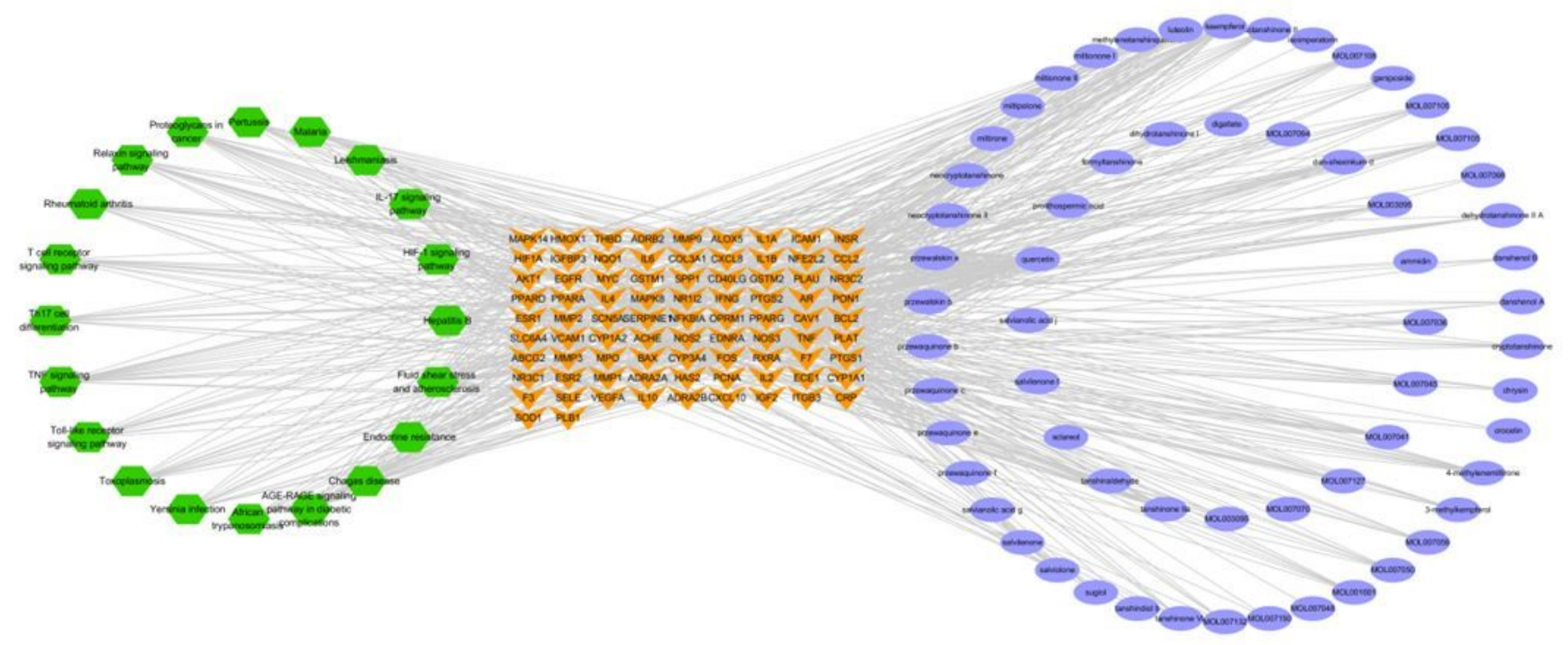

Figure 6

ZD-CHD network. Purple ellipse represents the putative therapeutic active ingredients of ZD, orange arrow represents the common targets from ZD and CHD, and green hexagon represents the KEGG pathways.

\section{Supplementary Files}

This is a list of supplementary files associated with this preprint. Click to download.

- Supplementarymaterials.docx

- Supplementarymaterials.docx 